\title{
ELECTROMAGNETIC, THERMAL, AND STRUCTURAL ANALYSIS OF RF CAVITIES USING ANSYS
}

\author{
N. Hartman, R. A. Rimmer; \\ Lawrence Berkeley National Laboratory, Berkeley, CA 94720, USA
}

\section{Abstract}

We report on techniques developed for producing electromagnetic, thermal, and structural solutions to RF cavity design problems in ANSYS, using one model [1]. Methods for preparing imported geometry from solid modeling programs are discussed, and meshing techniques are suggested. A study of mesh density is presented, comparing mesh size with heat flux and Q factor convergence. The general analysis protocol is presented in a stepwise fashion, describing the macros that are used for conducting RF calculations. Finally, these techniques are applied to a proposed RF cavity for the NLC damping rings, which is shown as an example.

\section{IMPORTING GEOMETRY}

Often a solid model of the desired cavity geometry will exist, and it is usually faster to import this geometry into ANSYS, rather than creating the geometry internally. Although some geometry manipulation within the Finite Element Analysis (FEA) model is generally required, it is always advantageous to keep this to a minimum. Importation may be accomplished using a transfer format, such as IGES, or by opening the native file directly into ANSYS, if the capability exists (such as for Pro/Engineer) [2]. In general, the solid model should be simplified as much as possible before transferring to ANSYS; this simplification may include suppressing extraneous features (such as bolt holes) as well as removing components that are not needed in the analysis (such as bolts). Also, the FEA model should make maximum use of symmetry conditions (although all symmetry planes should contain the beam axis).

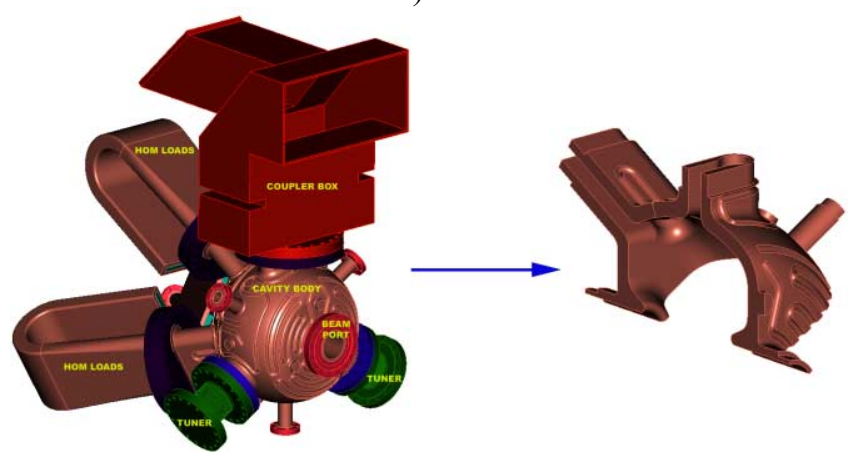

Figure 1. Cavity assembly in Pro/Engineer at left, with import geometry shown at right (after simplifying).

The solid model may be "cut" in order to create a symmetry section before or after importing into ANSYS, though it is usually faster to perform the cut in the solid modeler, as it reduces the size of the transfer file. Figure 1 shows the original solid model and the portion of the model that is imported into ANSYS for the Next Linear Collider (NLC) damping ring RF cavity design $[3,4]$.

After the cavity geometry has been imported, the vacuum volume must be created. This can be simply accomplished by "capping" the symmetry planes with areas, and then creating a volume. It is important that the vacuum volume shares its outer boundary with the inner boundary of the cavity, or the meshes between the two volumes will not be associated. Figure 2 shows the process of creating the vacuum volume within ANSYS.

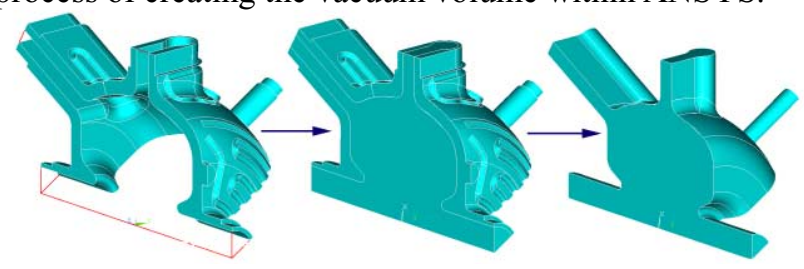

Figure 2. Using imported geometry to create the "vacuum" volume within ANSYS.

\section{MESHING}

Creating an acceptable mesh is an iterative process. Although it will later be shown that electromagnetic results are fairly insensitive to mesh size, surface heat flux is highly dependent on the mesh density at the vacuum boundary. For this reason, it is advantageous to create a fine mesh in critical areas on the surfaces (such as near high power HOM ports), while retaining a larger mesh in the body in order to reduce run time and memory usage. A simple way to achieve this mesh variation is to divide the vacuum volume into sub-volumes depending on the needed local mesh size. In this way, not only can the surface mesh be controlled by sizing areas and lines, but the "global" mesh size can be set on a local basis for each sub-volume, resulting in better mesh control (Figure 3).

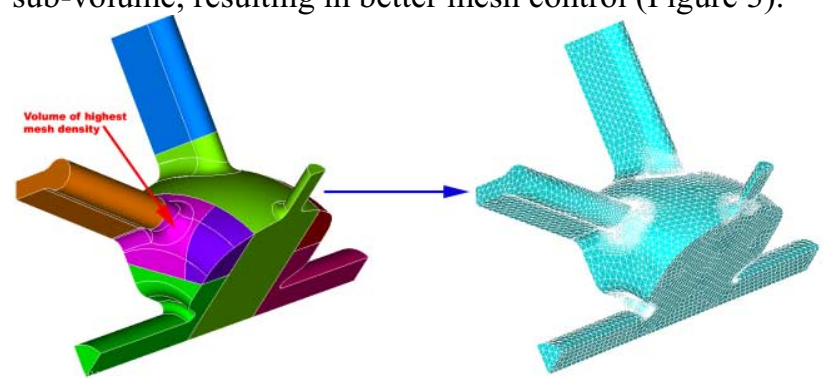

Figure 3. Vacuum volume divided for meshing, along with corresponding mesh of HF119 tetrahedra. 


\section{ELECTROMAGNETIC ANALYSIS}

After the HF119 mesh (or HF120, for brick models) has been created, the material properties are set and the boundary conditions defined. In ANSYS, high frequency electromagnetic analyses default to MKS units, and the properties of the resonating material are given as ratios to the free space permittivity and permeability (in this case vacuum is the material, so these properties are both 1).

Boundary conditions can be defined in two possible ways: first, as electric walls on the vacuum/cavity interface surfaces, or second, as electric walls with impedance conditions. Adding impedance boundary conditions allows ANSYS to calculate the cavity's Q internally, but requires that the surface resistance $\left(R_{S}\right)$ be defined initially:

$$
R_{S}=\frac{1}{\sigma \delta}=\sqrt{\pi f \mu_{o} / \sigma}
$$

$\sigma=$ conductivty $; \delta=$ skin-depth $\mu_{O}=$ permeabilty $; f=$ frequency

In all cases, at least the electric walls must be defined, or the analysis will not solve properly. Any surface that is not defined as an electric wall is considered by ANSYS to be a magnetic wall condition (Figure 4).

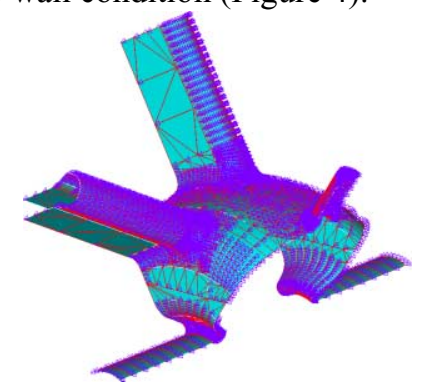

Figure 4. Vacuum/cavity interface surfaces shown with both electric wall and impedance boundary conditions.

Before issuing the solve command, the analysis type must be set to modal and the frequency range must be defined. Since the target frequency is usually fairly well known, the range should be limited. It is also important to specify that the modes be extracted, meaning that ANSYS calculates not just frequency, but also element results (if this is not done, there will be no $\mathrm{E}$ or $\mathrm{H}$ field data). $\mathrm{E}$ field and $\mathrm{H}$ field results for the NLC $700 \mathrm{MHz}$ cavity are shown in Figure 5. Note that the scale has been omitted by the author, as ANSYS normalizes all results to some arbitrary value; it is only the ratios of results that are quantitatively useful.

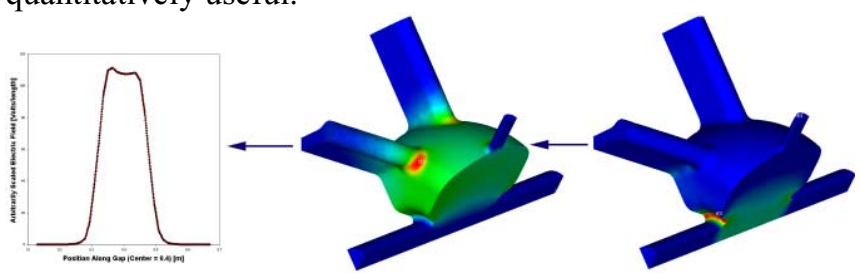

Figure 5. E field, $\mathrm{H}$ field, and $\mathrm{E}$ field profile along the beam axis, for the NLC cavity (right to left).

\section{CALCULATING SURFACE POWER LOSSES}

If impedance boundary conditions have been used in the electromagnetic analysis, ANSYS will calculate the total surface power losses when the "qfact" macro is issued. However, in order to find the distribution of power losses on the entire cavity surface, it is necessary to use surface effect elements and a post processing macro written to accomplish this. First, SURF152 elements are meshed over the cavity/vacuum boundary in order to exactly match the underlying volume mesh. (Note that the SURF152 elements must be specified without midside nodes and with heat flux loading capability.) A macro is then used to calculate the total power loss $\left(P_{S}\right)$ over the vacuum/cavity boundary:

$$
P_{S}=\frac{1}{2} \int_{S} H^{2} R_{S} d A
$$

$$
H=\text { magnetic- field } ; S=\text { boundary-surface }
$$

This total calculated power loss is then normalized to the expected power loss in the cavity and used as a scaling factor for applying heat flux loads on an element by element basis (over the surface elements) (Figure 6).

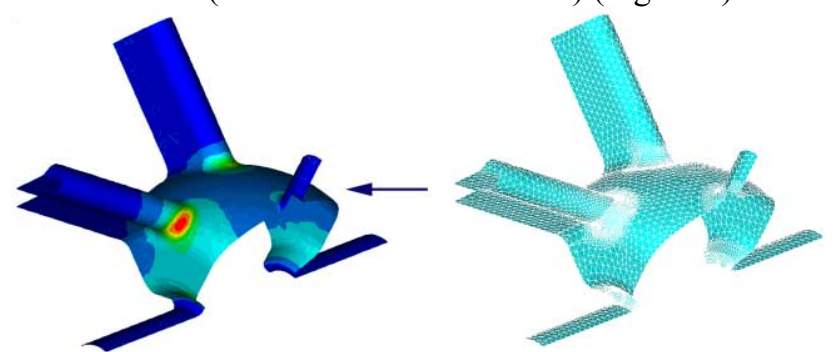

Figure 6. Surface element mesh (right) and applied heat flux loads (left). Scale has been omitted.

\section{ELECTROMAGNETIC QUANTITIES}

Typically it is desirable to calculate the cavity's quality and shunt impedance. These two parameters follow partially from the previous power loss calculation (Eq. 2), but also require calculating the total stored energy $(U)$ in the cavity:

$$
\begin{gathered}
U=\frac{1}{2} \int_{V} H^{2} \mu_{o} d V=\frac{1}{2} \int_{V} E^{2} \varepsilon_{o} d V \\
\text { E=electric- field } ; \varepsilon_{O}=\text { permittivity } ; V=\text { vacuum-volume }
\end{gathered}
$$

After the cavity voltage $(V)$ has been calculated by integrating the electric field along the beam axis (plot shown in Figure 5), the shunt impedance $\left(R T^{2}\right)$ and quality factor $(Q)$ follow:

$$
Q=2 \pi f \frac{U}{P_{S}} ; R T^{2}=\frac{V^{2}}{2 P_{S}}
$$

Note that the transit time factor has not been included here, but may be incorporated in the calculation if so desired. 


\section{THERMAL AND STRUCTURAL ANALYSES}

Once the heat flux loads have been applied and all desired electromagnetic quantities have been calculated, the HF119 or 120 mesh may be deleted. The cavity volume (rather than the vacuum) can now be meshed with thermal elements, and the heat flux loads from the surface elements will automatically transfer (as long as the initial area associations between the cavity and vacuum have not been removed). The thermal model can be constrained in any of several different ways; in the case of the NLC cavity, cooling channels were cut into the cavity model and convective boundary conditions were applied to the surfaces of these channels (Figure 7).
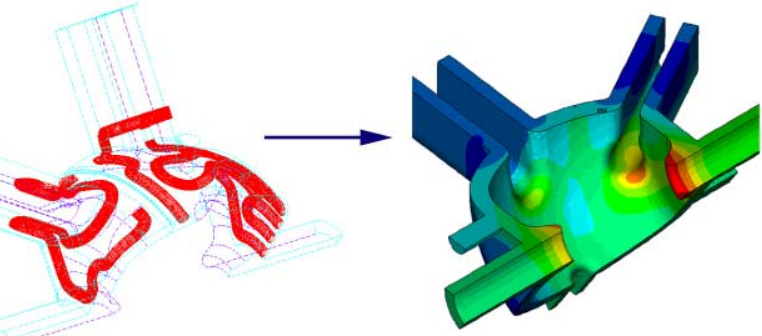

Figure 7. NLC cavity model showing cooling channels in wire-frame (left) and resulting thermal solution (right).

After obtaining the thermal solution, the structural solution may be calculated by applying the thermal analysis results as a load in the model. These temperatures may also be combined with other types of loading, such as vacuum and port loads. The model should be constrained as realistically as possible (in addition to the requisite symmetry constraints) since the fixation scheme may dominate the shape of the cavity and the stresses that it experiences. The NLC cavity is shown below with near kinematic constraints, inducing the least possible amount of stress (Figure 8).

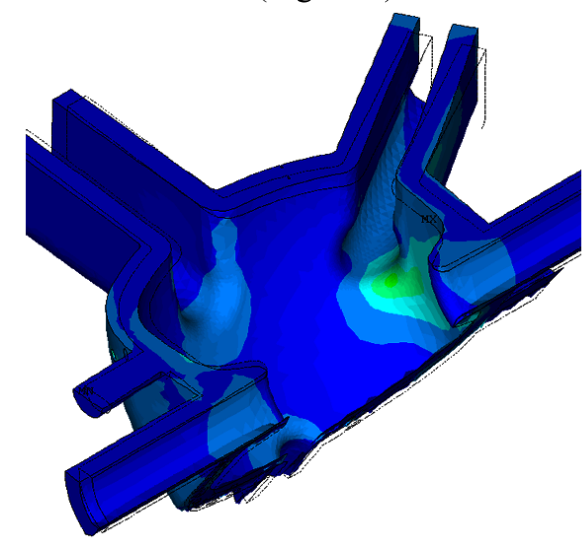

Figure 8. NLC cavity shown in displaced form, with contours indicating relative stress levels.

\section{MODELING RESULTS}

In order to compare the effectiveness and accuracy of ANSYS as a design tool for cavity development, the NLC design shown above was compared with a MAFIA model of identical geometry (to the extent possible given each program's restrictions) [5]. Additionally, two ANSYS models of different levels of mesh refinement were compared in order to determine the effects of mesh density. Results are shown in Table 1.

Table 1: ANSYS and MAFIA results compared for the NLC cavity design.

\begin{tabular}{|c|c|c|c|}
\hline Parameter & ANSYS 1 & ANSYS 2 & MAFIA \\
\hline \# Elements & 48808 & 84565 & N/A \\
\hline Q & 28384 & 28812 & 34609 \\
\hline RT $^{\mathbf{2}}$ (MOhms) & 3.46 & 3.45 & 4.06 \\
\hline Freq. (MHz) & 691 & 691 & 704 \\
\hline
\end{tabular}

\section{ADDITIONAL CONCERNS}

\subsection{Model Symmetry}

Since using symmetry is one of the easiest ways to reduce a model's size, run time, and memory needs, the effect of symmetry on accuracy was examined for a simple pillbox model. Several models were run, each one a portion of a complete pillbox. The largest model (least symmetry) was a 90 degree section, while the smallest was an 11.25 degree section. While the smallest to largest models differed in number of elements by an order of magnitude, less than a $1 \%$ decrease in accuracy was witnessed for both $\mathrm{Q}$ and shunt impedance calculations.

\subsection{Role of Mesh Size in Heat Flux Calculations}

It has already been mentioned that global electromagnetic quantities vary only slightly with large changes in mesh density; this is not the case, however, for local effects, such as maximum surface heat flux. Iterative testing has shown that "hot spot" heat fluxes may vary up to $20 \%$ with changes in mesh density. Since the highest stresses in a cavity occur at the hot spot, this effect can have ramifications for safety factors and other aspects of cavity design. Table 2 shows representative heat flux variations for the Pep II RF cavity design [6].

Table 2: Maximum heat flux versus mesh size.

\begin{tabular}{|c|c|c|c|c|}
\hline Element Size (mm) $>$ & 3.0 & 1.5 & 0.75 & 0.2 \\
\hline Heat Flux $\left(\mathbf{W} / \mathbf{c m}^{2}\right)>$ & 120 & 138 & 143 & 148 \\
\hline
\end{tabular}

\section{REFERENCES}

[1] ANSYS is a trademark of SAS Inc. www.ansys.com [2] Pro/Engineer is a trademark of PTC Inc. www.ptc.com [3] R. A. Rimmer, et al., "RF Cavity R\&D at LBNL for the NLC Damping Rings", LBNL \#47949.

[4] R. A. Rimmer, et al., "An RF Cavity for the NLC Damping Rings", PAC 2001 Proceedings, Paper MPPH064.

[5] MAFIA is a trademark of CST Inc. www.cst.de [6] R. A. Rimmer, et al., "PEP-II RF Cavity Revisited", LCC-0032, CBP Tech Note 197. 